\title{
Information Access Control of Privacy Patterns on Online Photo Sharing
}

\author{
M. Ashok Kumar'1, J. Albert Mayan² \\ 1Sathyabama University, Chennai, India. \\ 2Department of CSE, Sathyabama University, Chennai, India
}

\begin{abstract}
Innovation made mingling with people exceptionally basic and simple. Interfacing with people can be done with just a click of button today. The security of our own data and sharing that data in the advanced world has dependably been a noteworthy test for the always developing informal communities. Concerning the relationship in the midst of individuals and progression, the issue of trust is a factor of debate dependably. This paper endeavor to address this issue and study the situation when a client shares a photograph containing people other than himself or herself (termed co-photograph for short).To forestall conceivable protection spillage of a photograph, outline a component to empower every person in a photograph know about the posting action and partake in the photograph posting. For this reason, we require an effective facial acknowledgment (FR) framework that can perceive everybody in the photograph. Be that as it may, all the more requesting security setting may restrict the quantity of the photographs openly accessible to prepare the FR framework. To manage this issue, our component endeavors to use clients' private photographs to outline a customized FR framework particularly prepared to separate conceivable photograph co-proprietors without releasing their protection. We additionally build up a disseminated agreement based technique to diminish the computational multifaceted nature and secure the private preparing set. We demonstrate that our framework is better than other conceivable methodologies as far as acknowledgment proportion and productivity. Our component is actualized as a proof of idea Android application on Face book's stage.
\end{abstract}

Keywords: Co-photo; Online social network; Photo sharing; Privacy policy; Facial Recognition System.

\section{INTRODUCTION}

Online social network have ended up necessary piece of our everyday life what's more, has significantly changed the way we interface with one another, satisfying our social needs-the needs for social cooperation's, data sharing, appreciation what's more, regard. It is likewise this very nature of online networking that makes individuals put more substance, including photographs, over online social network without an excessive amount of thought on the substance. On the other hand, once something, for example, a photograph, is posted on the web, it turns into a lasting record, which may be utilized for purposes we never anticipate. For instance, a posted photograph in a gathering may uncover an association of a superstar to a mafia world. Since Online social networks end user may be inconsiderate in posting content while the impact is as such coming to, security assurance over social network turns into a critical issue. At the point when more capacities, for example, photograph sharing and labeling are included, the circumstance turns out to be more muddled. For example, these days we can share any photograph as we like on OSNs, paying little heed to whether this photograph contains other individuals (is a co-photograph) or not. As of now there is no limitation with sharing of co-photographs, on the opposite, interpersonal organization administration suppliers like Face book are urging clients to post co-photographs and tag their companions with a specific end goal to get more individuals included. On the other hand, imagine a scenario where the co-proprietors of a photograph are not willing to share this photograph? Is it a security infringement to share this co-photo without authorization of the co-proprietors? Should the co-proprietors have some control over the co-photograph? To answer these questions, we need to elaborate on the privacy issues over online social network. Traditionally, privacy is regarded as a state of social withdrawal.

\section{Related WoRK}

In [12], Mavridis et al. study the insights of photograph sharing on informal communities and propose a three domains display: "a social domain, in which 
personalities are elements, and fellowship a connection; second, a visual tactile domain, of which faces are substances, and co-event in pictures a connection; and third, a physical domain, in which bodies have a place, with physical nearness being a connection." They demonstrate that any two domains are profoundly corresponded. Given data in one domain, we can give a decent estimation of the relationship of the other domain.

The security and protection issues in online social networks likewise rise as critical and significant exploration subjects. The protection spillage brought about by the poor access control of shared information in Web. To manage this issue, access control plans are proposed in [13] and [4]. In these works, adaptable access control plans in light of social settings are researched. Be that as it may, in current online social networks, when posting a photograph, a client is not required to request authorizations of different clients showing up in the photograph.

Every client can characterize his/her protection strategy and introduction approach. Just when a photograph is handled with proprietor's protection strategy and co-proprietor's introduction arrangement could it be posted. Be that as it may, the co-proprietors of a co-photograph can't be resolved consequently; rather, potential co-proprietors must be recognized by utilizing the labelling elements on the current online social networks.

\section{PROBLEM STATEMENT AND HYPOTHESES}

\section{A. Privacy policy and exposure policy}

We consider that every end user $i$ has a privacy approach $\mathrm{Pi}(\mathrm{x})$ and an exposure strategy $\mathrm{Vi}(\mathrm{x})$ for a particular photograph $x$. The privacy approach $\mathrm{Pi}(\mathrm{x})$ shows the arrangement of clients who can get to photograph $\mathrm{x}$ and exposure strategy $\mathrm{Vi}(\mathrm{x})$ demonstrates the arrangement of clients who can get to $x$ when client $i$ is included. After individuals on co-photograph $x$ are perceived with our calculation as a set I, the arrangement of end user who take after both the privacy strategy and exposure approach could be computed.

We accept that our end users have characterized their privacy strategy and exposure approach and these arrangements are modifiable. The introduction arrangement is dealt with as private information that should not be uncovered, and a safe set crossing point convention is utilized to discover the access approach that is calculated.

\section{B. FR with social contexts}

A FR engine for an expansive scale interpersonal organization may require segregating a large number of people. It is by all accounts an overwhelming undertaking that could never be accomplished. Social settings contain a lot of valuable data which could be used as from the earlier learning to help the facial recognition. From three domain models, it is demonstrated that the relationship in the social domain and physical domain are exceedingly associated with the relationship in the visual tactile domain. In this way, we can utilize the social connection to build from the earlier circulation over the characters on the co-photographs for client. With this priori appropriation, while attempting to perceive individuals on the co-photographs, the FR motor could concentrate on a little partition of close, companions who are geologically close and collaborating much of the time with client.

\section{FR system}

We accept that user has a photograph set of himself/herself as his/her private preparing tests (say, put away on his/her own gadget, for example, advanced mobile phone). From the private photograph set, a client distinguishes and extricates the appearances on every photograph with the standard face identification technique. For every face, a vector of size $p$ is separated as the component vector. With the private preparing set, every client will have a individual FR engine to recognize his/her one-jump neighbors. The individual FR can be built as a multi-class order framework, where every class is relating to one client (himself/herself or one companion).

One-against-all system utilizes champ take-all strategy. It develops $n$ parallel classifiers for each of $n$ classes. The objective of every double classifier is to recognize one class from the rest with a choice capacity.

One-against-one technique utilizes max-voting-win procedure. It builds $\mathrm{n}$ paired classifiers, in which every classifier is planned to recognize two classes. The thought is that in the event that we can recognize any two 
classes, then we can distinguish any of them. Thus, classifier uij is developed by taking records from i as positive examples and records from $\mathrm{j}$ as negative ones.

\section{SyStem OVERVIEW}

Here, we exhibit the point by point portrayal of our framework. As a rule, the agreement result could be accomplish by iteratively refining the neighborhood preparing result: firstly, every client performs nearby regulated learning just with its own particular preparing set, then the neighborhood results are traded among colleagues to frame a worldwide learning. In the next round, the worldwide learning is utilized to regularize the nearby preparing until meeting. In this area, firstly, we utilize a toy framework with two clients to exhibit the standard of our configuration. At that point, we examine how to construct a general individual FR with more than two clients. At last, we examine the adaptability of our configuration at the extensive size of OSNs.

\section{A. A toy system}

Assume there are just two clients user1 and user2 with private preparing information $x 1$ and $x 2$. To recognize them, we just need to locate a binary decision function. For the most part, the proposed disseminated preparing plan of a toy framework could be outlined in Algorithm which uses iterative method to compute classifiers. Threshold is the user-defined stopping criteria, a larger threshold results with fewer iterations while a larger discrepancy between positive and negative samples.

\section{B. Online social networks with social contexts}

In the past subsection, we demonstrate to manufacture a twofold classifier in a toy framework with two clients. At the point when Considering the functional situation, every client may have more than one companion, and along these lines multi-class classifiers are required. As a rule, a multi-class classifier is accomplished by utilizing one of the two systems to join a few parallel classifiers: one-against-all and one-againstone. In this area, we dissect their execution furthermore, give components the correct technique.

\section{Two strategies and classifier reuse}

The methodology of one-against-all, every client are connected with a two binary classifier by making initiator and cooperators. In examination, classifiers can't be reused in the one-against-all methodology in light of the fact that they are prepared with diverse cooperators. As per Classifier Computation Algorithm, there are two stages to fabricate classifiers for every area: firstly discover classifiers of fself, friendg for every hub, then discover classifiers of ffriend, friendg. Notice that the second step is precarious, since the companion rundown of the area proprietor could be uncovered to all his/her companions. Then again, companions may not know how to speak with each other. For this thought, when building classifiers of ffriend, friendg, all the nearby preparing results are send to the area proprietor, who will facilitate the communitarian forwarding so as to prepare procedures nearby preparing results to right associates. In this way, companions need not to know who they are working with what's more, how to converse with them.

\section{4.2.2 Stranger detection}

At the point client can separate his entire companion with classifiers. The main thing remains to gather double classifiers to be a multi-class classifier. In this paper, we build a choice tree by orchestrating double classifiers comparably to the DAGSVM.

\section{PERFORMANCE ANALYSIS}

\section{A. Benefits of our design}

Centralized approach has brought together FR engine responsible for perceiving all clients over a vast OSN. To secure the preparation photographs, a protection saving SVM preparing strategy is utilized. Oneagainst-all methodology decays the fellowship chart and utilizes our proposed accord based preparing strategy to perform synergistic preparing. The development of the new system contains the activities, which try to automate the entire process keeping in view of the database integration approach. One-against-one analysis approach is similar to the one-against-all approach, except that the average rounds in one training process should be much less, due to the fact that there are only two participants. If we consider the complete sub graph in the friendship graph, the expected cost should be less than the other models. 


\section{B. $\quad 5.2$ Security analysis}

Access policy is determined by the intersection of owner's privacy policy and co-owners' exposure policy.

\section{EVALUATION}

Our framework is assessed with two criteria: system wide execution and facial acknowledgment execution. The previous is utilized to catch this present reality execution of our outline on expansive scale OSNs as far as calculation taken a toll, while the recent is an imperative component for the client experience. In this segment, we will depict our Android execution first and afterward the investigations to assess these two criteria.

\section{A. Implementation}

Our model works in three modes: a setup mode, a resting mode and a working mode. Running in the setup mode, the project is working towards the foundation of the choice tree. For this reason, the private preparing set $\mathrm{Xi}$ and neighborhood $\mathrm{Bi}$ should be indicated. Xi could be indicated by the client with the catch "Private preparing set". When it is squeezed, photographs in the PDA exhibitions could be chosen and added. To setup the area at this stage, a client needs to physically determine the arrangement of "dear companions" among their Facebook companions with the catch "Pick companions" as their neighborhood.

Amid the preparation handle, an attachment is set up trade nearby preparing results. After the classifiers are acquired, choice tree is built and the project changes from the setup mode to the resting mode. Facebook permits us to make a rundown of companions, for example, "dear companions" or "Colleagues". We can share a photograph just to companions on rundown. As indicated by the proposed plan, this companion rundown ought to be convergence of proprietor's security arrangement and co-proprietors' presentation approaches. Be that as it may, in Facebook API, companion records are perused just things, they can't be made or upgraded through the current Programming interface. That implies we can't tweak a companion list to share a co-photograph. At present, when the catch "Post Photograph" is squeezed, co-proprietors of $\mathrm{x}$ are distinguished, then warnings alongside $x$ are send to the co-proprietors to demand consents. In the event that they all consent to post $\mathrm{x}, \mathrm{x}$ will be shared on the proprietor's page like an ordinary photograph. In this sense, clients could indicate their security approach yet their presentation strategies are either everyone on earth or no one contingent upon their mentality toward $x$. The information stream for a photograph posting action is delineated by the strong red bolts. After the solicitations are conveyed, the system will backpedal to the dozing mode. In the event that $\mathrm{Xi}$ or $\mathrm{Bi}$ is changed, the system will be conjured to the setup mode. In this case, the operations in the yellow dashed box will be performed again and choice tree will be redesigned.

\section{B. Facial recognition performance}

We demonstrate that acknowledgment proportions of our proposed plan and the plan with DAG choice tree. When there are no outsiders, both our proposed plan and the DAG plan could accomplish high acknowledgment proportion of more than $80 \%$ when the number of clients is less than 30 . While in among the clients, $10 \%$ of them are outsiders, we can see that the acknowledgment proportion of our plan has a higher acknowledgment proportion than the DAG plan by $5 \%$. The reason is that our plan can dismiss outsiders. The strong line on every figure speaks to acknowledgment proportion of outsiders, which is expanding with number of clients. Naturally, if there are more clients, there will be more classifiers and the chance that an outsider gets conflicting choices will be higher. When there are $30 \%$ outsiders, our plan beats the DAG plan by $10 \%$ in terms of acknowledgment proportion. This is accomplished by the capacity of distinguishing outsiders. With 30 clients, the likelihood of distinguishing an outsider is around $35 \%$.performed again and choice tree will be redesigned. 


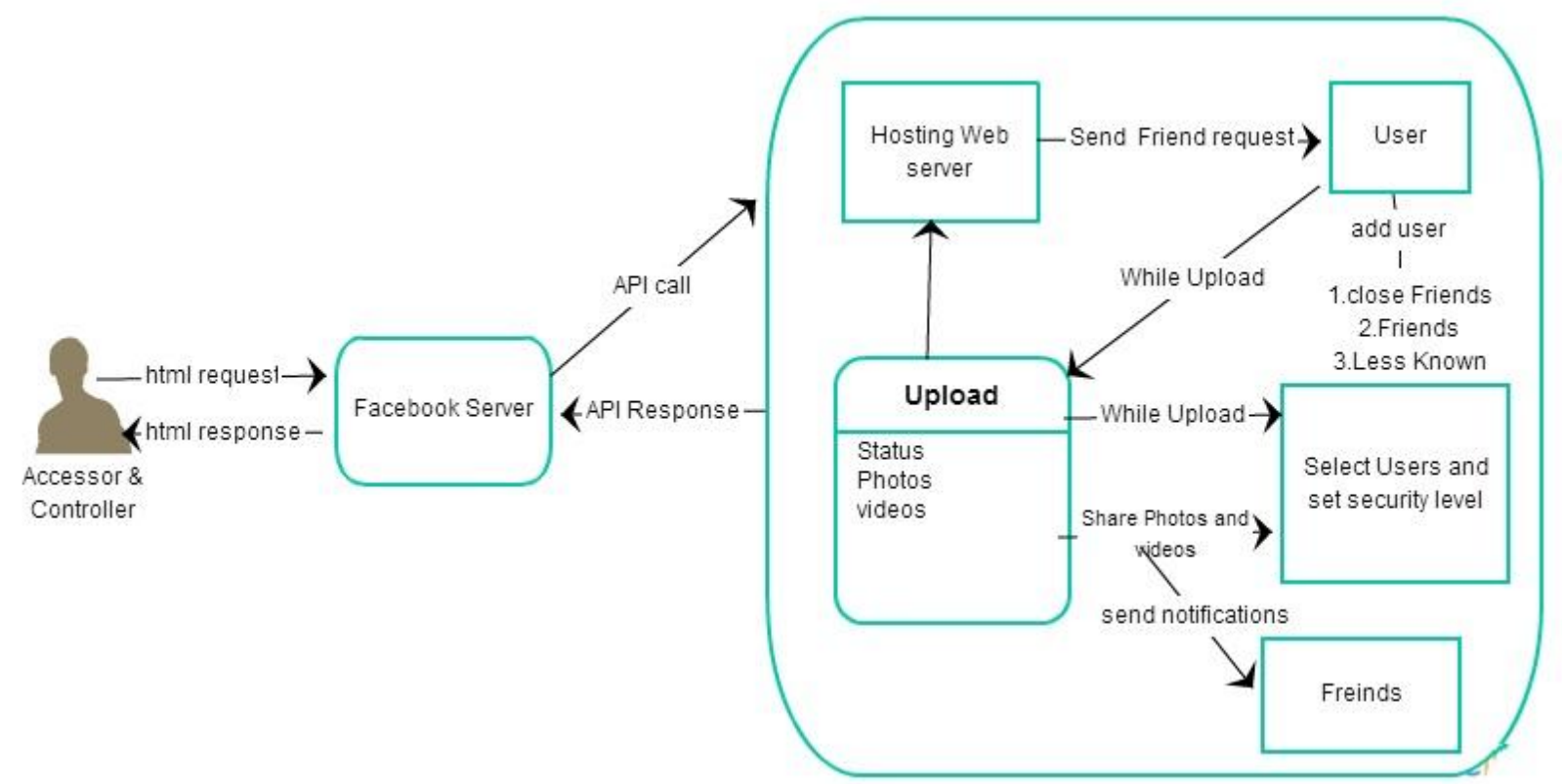

Fig. 1: System structure of our application

\section{CONCLUSION}

Photograph sharing is a standout amongst the most prevalent elements in online informal communities, for example, Facebook. Tragically, inconsiderate photograph posting may uncover protection of people in a posted photograph. To check the protection spillage, we proposed to empower people conceivably in a photograph to give the authorizations before posting a cophotograph. We composed a protection saving FR framework to recognize people in a co-photograph. The proposed framework is included with low calculation expense and privacy of the preparation set. Hypothetical investigation and tests were directed to show adequacy and productivity of the proposed plan. We expect that our proposed plan be exceptionally valuable in securing clients' protection in photograph/picture sharing over online informal organizations. In any case, there dependably exist exchange off in the middle of protection and utility. For instance, in our present Android application, the co-photograph must be post with authorization of all the co-proprietors. Inactivity presented in this procedure will significantly affect client experience of OSNs. Moreover, neighbourhood FR preparing will deplete battery rapidly. Our future work could be the means by which to move the proposed preparing plans to individual mists like Dropbox and/or icloud.

\section{REFERENCES}

[1] Besmer and H. Richter Lipford. Moving beyond untagging: photo privacy in a tagged world. In Proceedings of the SIGCHI Conference on Human Factors in Computing Systems, CHI '10, pages 1563-1572, New York, NY, USA, 2010. ACM.

[2] S. Boyd, N. Parikh, E. Chu, B. Peleato, and J. Eckstein. Distributed optimization and statistical learning via the alternating direction method of multipliers. Found. Trends Mach. Learn., 3(1):1-122, Jan. 2011.

[3] L. Palen. Unpacking privacy for a networked world. pages 129- 136. Press, 2003.

[4] Z. Stone, T. Zickler, and T. Darrell. Toward large-scale face recognition using social network context. Proceedings of the IEEE, 98(8):1408-1415

[5] Acronymics Inc. AgentBuilder. http://agentbuilder.com/.

[6] K.-B. Duan and S. S. Keerthi. Which is the best multiclass svm method? an empirical study. In Proceedings of the 6th international conference on Multiple Classifier Systems, MCS'05, pages 278-285, Berlin, Heidelberg, 2005. Springer-Verlag .

[7] L. Kissner and D. X. Song. Privacy-preserving set operations. In V. Shoup, editor, CRYPTO, volume 3621 of Lecture Notes in Computer Science, pages 241-257. Springer, 2005. 